\title{
Analyzing the Rare Earth Elements (REE's) and Trace Metals in Tailings
}

Rachel Butler ${ }^{1}$, Deepak Pudasainee ${ }^{1}$, Md Khan $^{1}$, Rajender Gupta ${ }^{1}$

${ }^{1}$ Department of Chemical and Materials Engineering, University of Alberta

\begin{abstract}
In the process of producing bitumen from oil sand, a by-product called tailings is produced. Tailings are a mixture of clay, fine particles, water, solvent and residual bitumen. The industry's current approach is to leave them in tailings ponds; however, that may cause environmental impacts to the ecosystems around them due in part to the toxic trace metals found in them. Research has shown that there are also valuable rare Earth elements (REEs) present in tailings. REEs found in tailings include Cerium, Neodymium, Lanthanum etc. Iron, Titanium, and Zirconium are not considered REEs but are still valuable enough to be extracted. The objective of this research was to determine the concentration of REEs and trace metals in bitumen froth treatment tailings (FTT). Our research team used acid digestion and inductively coupled plasma mass spectroscopy (ICP-MS) to measure the concentration of REEs and trace metals in several samples of FTT ash. We learned that Cerium was the most prevalent REE in tailings samples (>1000ppm), followed by Neodymium and Lanthanum. Zirconium was the most prevalent trace metal found in this tailings sample $(>1000 \mathrm{ppm})$, followed closely by Vanadium. Knowing the exact concentration of harmful trace metals in tailings will allow us to determine the extent of tailings ponds environmental effect and toxicity. Collecting and selling expensive metals found in tailings could be the start of a new precious metals economy in Alberta, which would provide new investment opportunities and jobs. This would also encourage corporations to invest in finding new ways to extract these precious metals, resulting in more purified tailings and less tailings overall going into tailings ponds.
\end{abstract}

Key words:

bitumen, froth treatment tailings, Gupta lab, rare earth elements, trace metals, oil sands, TGA, alberta, chemical engineering

Cite as: Butler R., Pudasainee D., Khan M., Gupta R. 2019. Analyzing the rare earth elements (REEs) and trace metals in tailings. Alberta Academic Review, Vol 2 (2) 17-18, WISEST Special Issue (non peer-reviewed), DOI 10.29173/aar54. 


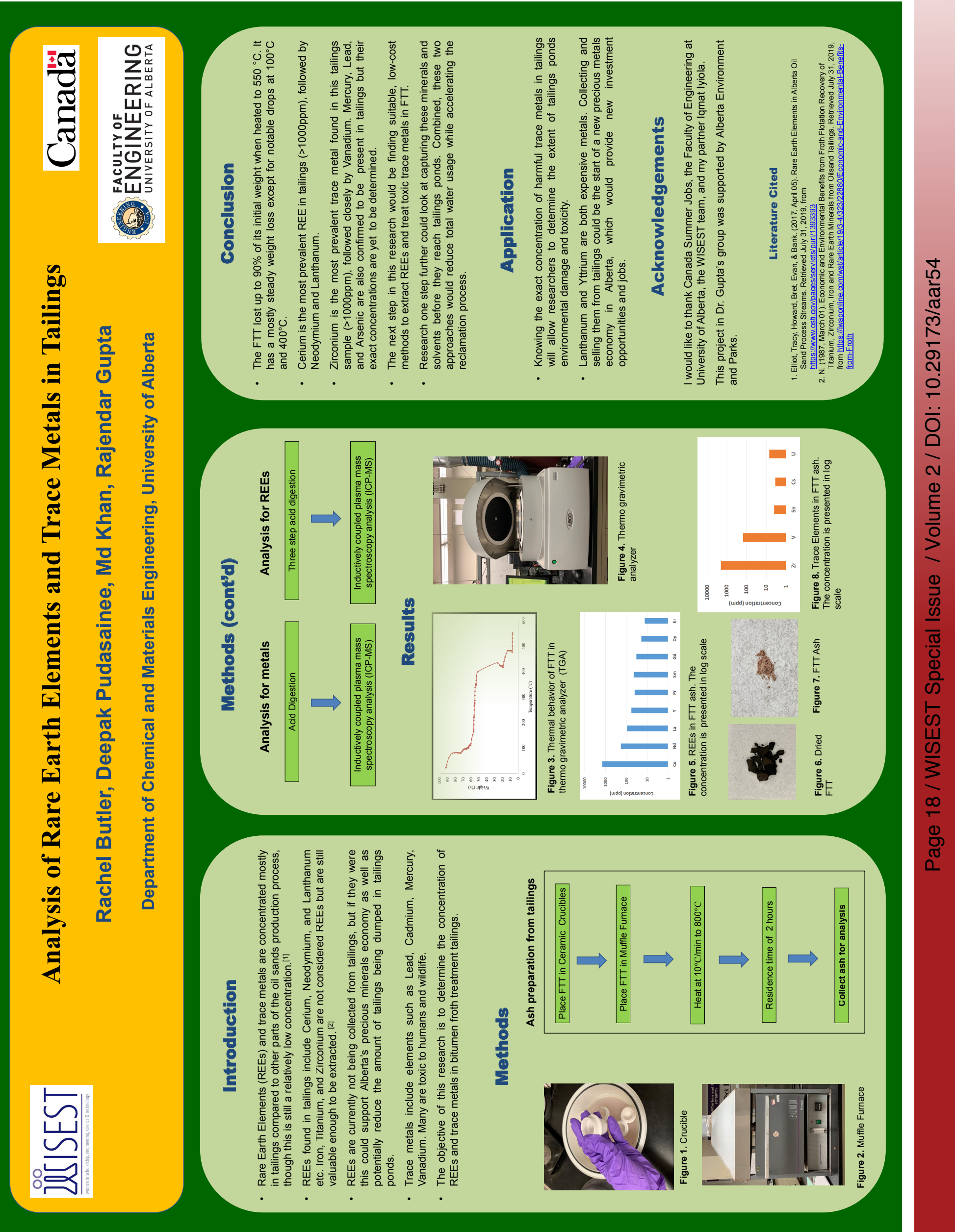

